\title{
Chaos in Neural Networks at Nonlinear Synapses
}

\author{
Algis Garliauskas, Remigijus Andžius \\ Department of Neuroinformatics \\ Institute of Mathematics and Informatics \\ Akademijos 4, 2600, Vilnius, Lithuania
}

Neural networks as massive parallel connectionist structures with nonlinearities are able to different complex phenomena as a self-organizing, associativity, bifurcation, and chaos.

It is well known an universal theory of behavior in nonlinear systems based on Los Alamos laboratory in 1971. For the large scale functions at increasing of the parameter a distruction of stable cycle is happened and a cycle of period-doubling is occured. The doubling of a period is continued until the endlessness. In this paper we pay an attention to different nonlinearities which take place on the interconnections between neurons, i.e., the functions of an activity on the input of neuron which rely through synaptic strength to output of another neuron. Using the Hebb's rule of learning, a mathematical model has been constructed on behalf slow and fast equations with different modifications of nonlinear synapses as follows:

The slow equation:

$$
w_{i j}(k+1)=\left(1-D_{w}\right) w_{i j}(k)+\eta a_{i}(k) a_{j}(k)
$$

where $w_{i j}(k)$ is the weight (synaptical strength) between $i$ and $j$ neurons for the $k$ th recursion step, $a_{i}, a_{j}$ are potentials of $i$ and $j$ neurons, $D_{w}$ is a decay parameter of the weights, $\eta$ notates the rate of learning.

The fast equation:

$$
a_{i}(s+1)=\left(1-D_{a}\right) a_{i}(s)+E a_{\Sigma}(s)\left(1 \pm a_{i}(s)\right),
$$

where $a_{\Sigma}(s)=\sum_{l}\left[a_{i l}(s) w_{i l}(s)\right]+I_{i}(s), I_{i}(s)$ is the external input of neuron $i$ for $s$ th iteration step, $D_{a}$ is the decay parameter of potentials, $E$ is the excitatory parameter, $a_{i l}(s)=q\left(a_{l}(s)\right)$, i.e., there is nonlinear potential postsynaptic function from the potential of output (presynaptic potential) of the lth neuron. In the last member of Equation 2 the sign plus is taken if $a_{\Sigma}(s) \leqslant 0$, and minus if $a_{\Sigma}(s)>0$. These conditions are expressed a bipolar sigmoid function of neuron.

The synaptic function $g\left(a_{l}(s)\right.$ ) (further $g(x)$ ) reflects the mutual activity among presynaptic and postsynaptic potentials or a result of complex synapse-dendrite activation.

A membrane characteristic of a dendrite is a nonlinear (with two stable areas and one unstable) $N$-shaped form. The approximation was taken:

$$
g(a)=\left[x^{3}-3 x^{2}+3 x\left(1-c^{2}\right)\right] \cdot\left[3\left(1-c^{2}\right)\right]^{-1}
$$

under the conditions:

$$
g= \begin{cases}1 & \text { if } g(x)>1 \\ g(x) & \text { if } g(x)<-1 \\ -1 & \end{cases}
$$

where $\left[h_{0}, h_{1}\right],\left[h_{2} \leqslant x\right],\left[h_{1}, h_{2}\right], h_{0}, h_{1}$, and $h_{2}$ are restrictions of stable and unstable areas.

It was shown that the coupling strength nonlinearities between neurons in a neural network allow to obtain rich and complex bifurcation diagrams given some opportunities in recognizing of images. 